\title{
Farmacoeconomia aplicada ao tratamento medicamentoso para a COVID-19 em um hospital campanha
}

\author{
Pharmacoeconomics applied to drug treatment for COVID-19 in a campaign hospital
}
Farmacoeconomía aplicada al tratamiento farmacológico del COVID-19 en un hospital de campaña

Carina da Costa Braúna ${ }^{1 *}$, Paulo Monteiro Araujo1, Rossicleia Dias Carvalho², Maria das Graças Freire de Medeiros Carvalho ${ }^{1}$, Lívio Cesar Cunha Nunes ${ }^{1}$.

\begin{abstract}
RESUMO
Objetivo: Analisar a terapia farmacológica e os custos diretos envolvidos no tratamento da COVID-19 observados em um hospital de campanha. Métodos: Foi realizado um levantamento acerca do protocolo clínico adotado por um hospital campanha no estado do Piauí - Brasil, analisando-o segundo os custos e as indicações clínicas dos medicamentos propostos para tratamento farmacológico de COVID-19. Resultados: O protocolo estudado previa o uso de heparina, omeprazol, hidroxicloroquina, ceftriaxona, azitromicina, oseltamivir, ivermectina e metilprednisolona (Fase $2 \mathrm{a}$ ou $2 \mathrm{~b}$ ). Os medicamentos que representaram maior impacto no custo diário foram a ceftriaxona $R \$ 77,00(32,19 \%)$, omeprazol $R \$ 28,80(12,04 \%)$, heparina [Fases 1 e $2 a R \$ 32,19(13,46 \%)$ - Fase $2 b \mathrm{R} \$ 64,38(26,91 \%)]$ e a metilprednisolona $\mathrm{R} \$ 44,80$ (18,73\%). Foi observado o uso de dois medicamentos offlabel, a ivermectina e a hidroxicloroquina. Conclusão: O protocolo medicamentoso para COVID-19 utilizado no hospital campanha estudado, mostrou-se bem oneroso frente ao financiamento pretendido. Espera-se que avaliações farmacoeconômicas como a realizada por esse estudo permitam o melhor desenho de novas versões de protocolos com melhores relações de custo-benefício.
\end{abstract}

Palavras-chave: Custos e análise de custo, Infecções por coronavirus, Uso Off-Label.

\section{ABSTRACT}

Objective: Analyze pharmacological therapy and the direct costs involved in the treatment of COVID-19 observed in a field hospital. Methods: A survey was carried out on the clinical protocol adopted by a campaign hospital in the state of Piauí - Brazil, analyzing it according to the costs and clinical indications of the drugs proposed for pharmacological treatment of COVID-19. Results: The studied protocol proposed the use of heparin, omeprazole, hydroxychloroquine, ceftriaxone, azithromycin, oseltamivir, ivermectin and methylprednisolone (Phase $2 \mathrm{a}$ or $2 \mathrm{~b}$ ). The drugs that had the greatest impact on daily costs were ceftriaxone $R \$ 77.00(32.19 \%)$, omeprazole $R \$ 28.80$ $(12.04 \%)$, heparin [Phases 1 and $2 a \mathrm{R} \$ 32.19$ (13,46\%) - Phase $2 b \mathrm{R} \$ 64.38(26.91 \%)$ ] and methylprednisolone $R \$$ $44.80(18.73 \%)$. The use of two off-label drugs, ivermectin and hydroxychloroquine, was observed. Conclusion: The drug protocol for COVID-19 used in the campaign hospital studied, proved to be expensive in view of the intended financing. It is expected that pharmacoeconomic assessments such as the one carried out by this study will allow the best design of new versions of protocols with better cost-benefit ratios.

Keywords: Costs and cost analysis, Coronavirus infections, Off-Label Use.

\section{RESUMEN}

Objetivo: Analizar la terapia farmacológica y los costos directos involucrados en el tratamiento de COVID-19 observado en un hospital de campaña. Métodos: Se realizó una encuesta sobre el protocolo clínico adoptado por un hospital de campaña en el estado de Piauí - Brasil, analizándolo según los costos e indicaciones clínicas de los fármacos propuestos para el tratamiento farmacológico del COVID-19. Resultados: El protocolo estudiado preveía el uso de heparina, omeprazol, hidroxicloroquina, ceftriaxona, azitromicina, oseltamivir, ivermectina y metilprednisolona (Fase $2 \mathrm{a}$ o $2 \mathrm{~b}$ ). Los medicamentos que tuvieron mayor impacto en los costos diarios fueron ceftriaxona $R \$ 77,00(32,19 \%)$, omeprazol $R \$ 28,80(12,04 \%)$, heparina [Fases 1 y $2 a \mathrm{R} \$ 32,19(13,46 \%)$ - Fase $2 \mathrm{~b} R \$ 64,38(26,91 \%)$ y metilprednisolona $\mathrm{R} \$ 44,80(18,73 \%)$. Se observó el uso de dos fármacos fuera de etiqueta, ivermectina e hidroxicloroquina. Conclusión: El protocolo de medicamentos para COVID-19 utilizado en el hospital de campaña estudiado resultó ser muy costoso en vista del financiamiento previsto. Se espera que evaluaciones farmacoeconômicas como la realizada por este estudio permitan el mejor diseño de nuevas versiones de protocolos con mejores relaciones costo-beneficio.

Palabras clave: Costos y análisis de costo, Infecciones por coronavirus, Uso Fuera de lo Indicado.

${ }^{1}$ Universidade Federal do Piauí, Teresina - PI. *E-mail: carinabrauna@hotmail.com

${ }^{2}$ Hospital de Campanha Estadual Dirceu Arcoverde, Teresina - PI.

SUBMETIDO EM: 11/2020 


\section{INTRODUÇÃO}

A família dos coronavírus (CoV) é composta por 4 genogrupos; 1 (alpha), 2 (beta), 3 (Gamma) e 4 (delta), podendo infectar aves e mamíferos e causar diversos tipos de síndromes respiratórias (SHEAHAN TP, et al., 2017). Recentemente, no final de 2019, Wuhan, um emergente centro de negócios localizado na China, passou por um surto de um novo coronavírus que matou mais de 1.800 e infectou mais de 70 mil indivíduos nos primeiros 50 dias da epidemia. Esse vírus foi relatado como membro do subgrupo beta dos coronavírus e foi denominado pelo Comitê Internacional de Taxonomia de Vírus (ICTV) de SARS-CoV-2 e a doença de COVID-19 (SHEREEN MA, et al., 2020).

Siddiqi HK e Mehra MR (2020), propuseram para COVID-19, uma classificação em três estágios: 1 - início da infecção (febre, dor de cabeça, tosse seca); 2 - Fase pulmonar (2a - dificuldade para respirar sem hipóxia, imagens pulmonares com alterações, $2 b$ - (Hipóxia) e; 3 - Fase hiperinflamatória (tempestade de citocinas, IRHA (insuficiência Respiratória Hipoxêmica Aguda), IRA (Insuficiência Respiratória Aguda) e disfunção orgânica múltipla). A preocupação com o custo da terapia ainda se agrava mais quando é considerado que cerca de $55 \%$ dos pacientes apresentam sintomas leves a moderados (fase 1 ) e 10\%, sintomas graves (fases $2 a$ e $2 b$ ), existindo a possibilidade de pacientes transitarem entre diferentes estágios da doença e aumentar consideravelmente o tempo de permanência hospitalar (podendo durar meses). Um fator que tem uma relação direta com os custos envolvidos na terapia farmacológica (HUGG-UNIRIO, 2020).

Diante desse cenário, muitos sistemas de saúde entraram em colapso e falharam em absorver a demanda de pacientes pela eficiência de transmissão/infecção do SARS-CoV-2 observada em vários países, e, pela velocidade com que casos incidentes da doença buscam as unidades de saúde e hospitais para atendimento. Um número significativo de pessoas necessita cuidados hospitalares com internação em enfermaria ou unidade de terapia intensiva (UTI). O isolamento social tem sido recomendado para o controle da transmissão viral e o achatamento da curva epidemiológica, com o objetivo principal de oportunizar aos hospitais ter condições de absorver e tratar os doentes ao longo do tempo (BARBIRATO DS, et al., 2020)

Nesse ínterim, é importante frisar que até o momento não existe intervenção farmacológica com efetividade e segurança comprovada para a COVID-19 (FALAVIGNA M, et al., 2020; SANDERS JM, et al., 2020), devendo o uso de novas medicações ser reservado a utilização em contexto de pesquisa clínica (FALAVIGNA M, et al., 2020). Nessa situação o uso de medicamento off-label, ou seja, quando sua administração possui resultados sugestivos, todavia ainda não possui evidência bem estruturada de sua eficácia (TILLU G, et al., 2020), pareceu uma estratégia eficiente.

As avaliações farmacoeconômicas são uma das formas de avaliar a eficácia de intervenções, como um determinado produto farmacêutico ou estratégia de tratamento, fornecendo informações que permitem a alocação ideal de recursos limitados do sistema de saúde. Além disso, os estudos farmacoeconômicos consideram simultaneamente entradas (ou seja, "custos") e resultados (ou seja, "benefícios" clínicos) resultantes do tratamento de doenças (WALLEY T e HAYCOX A, 1997).

Considerando o cenário descrito, este estudo teve por objetivo inicial sintetizar a terapia e os custos diretos envolvidos no tratamento farmacológico da COVID-19 observados em um hospital campanha do estado do Piauí - Brasil. Posteriormente, foi realizada uma análise sobre a indicação dos medicamentos previstos no protocolo da instituição e suas possíveis justificativas para o uso no tratamento da infecção.

\section{MÉTODOS}

Foi realizado um levantamento acerca do protocolo clínico para o tratamento farmacológico de COVID-19 adotado por um hospital campanha localizado no estado do Piauí - Brasil. O estudo não foi avaliado pelo sistema do Comitê de Ética em Pesquisa (CEP)/Conselho Nacional de Ética em Pesquisa (CONEP) por tratarse de uma pesquisa ao qual objeto de estudo é o protocolo do tratamento e não o paciente.

Os medicamentos utilizados pelos pacientes internados na instituição foram avaliados segundo os custos e as indicações clínicas. Para estimativa dos custos de tratamento medicamentoso, considerou-se os seguintes fatores. 
Primariamente, os custos diários do tratamento foram estimados seguindo os regimes terapêuticos da terapia farmacológica proposta pelo protocolo clínico adotado pelo hospital para o tratamento da COVID-19. Para aqueles medicamentos com doses por peso considerou-se um paciente com $60 \mathrm{Kg}$ e não foi considerado o reaproveitamento de dose anterior fracionada.

Posteriormente, os valores foram baseados em preço de venda dos medicamentos de referência, retirados da tabela "preços máximos de medicamentos por princípio ativo" ANVISA (2020a) da secretaria executiva da Câmara de Regulação do Mercado de Medicamentos - CMED, com ICMS (Imposto sobre Circulação de Mercadorias e Serviços) de 18\%.

As indicações dos medicamentos foram determinadas por dois farmacêuticos com auxílio do bulário eletrônico ANVISA (2020b) da ANVISA (Agência Nacional de Vigilância Sanitária) e da enciclopédia farmacêutica Drugs.com (DRUGS.COM, 2020). Utilizou-se a sintomatologia prevista na classificação proposta por Siddiqi HK e Mehra MR (2020) para a COVID-19, na avaliação do tipo de indicação: label (previsto uso em bula) ou off-label (não previsto em bula). Por fim, para todos os medicamentos utilizados foi realizado uma sucinta discussão da justificativa de seu uso.

\section{RESULTADOS}

Os medicamentos propostos no protocolo clínico do hospital de campanha para terapia farmacológica de COVID-19 nas fases inicial (1) e pulmonar (2a e 2b), de acordo com a classificação de Siddiqi HK e Mehra MR (2020) foram: heparina, omeprazol, hidroxicloroquina, ceftriaxona, azitromicina, oseltamivir, ivermectina, metilprednisolona acrescidos pelos medicamentos em caso de necessidade dipirona, metoclopramida, glicose e insulina regular. Os esquemas terapêuticos dos medicamentos utilizados no tratamento da infecção encontram-se na Tabela 1.

Tabela 1 - Resumo do esquema terapêutico proposto pelo protocolo do hospital de campanha estudado para o tratamento farmacológico da COVID-19.

\begin{tabular}{|c|c|c|c|c|}
\hline \multirow{2}{*}{ Medicamento } & \multirow{2}{*}{ Duração } & \multirow{2}{*}{$\begin{array}{c}\text { Início } \\
\text { Fase 1 }\end{array}$} & \multicolumn{2}{|c|}{ Fase Pulmonar } \\
\hline & & & Fase 2A & Fase 2B \\
\hline Heparina SC $5000 \mathrm{Ul} / 0,25 \mathrm{~mL}$ & $\begin{array}{l}\text { Durante a } \\
\text { internação }\end{array}$ & 5000 UI 8/8h & 5000 UI 8/8h & $10000 \mathrm{UI} 8 / 8 \mathrm{~h}$ \\
\hline Omeprazol EV 40mg & $\begin{array}{l}\text { Durante a } \\
\text { internação }\end{array}$ & $40 \mathrm{mg} \mathrm{24/24h}$ & $40 \mathrm{mg} \mathrm{24/24h}$ & $40 \mathrm{mg} \mathrm{24/24h}$ \\
\hline Hidroxicloroquina VO 400mg & 7-10 dias & $400 \mathrm{mg} \mathrm{12/12h}$ & $400 \mathrm{mg} \mathrm{12/12h}$ & $400 \mathrm{mg} \mathrm{12/12h}$ \\
\hline Ceftriaxona EV 1g & 5-7 dias & $2 \mathrm{~g} \mathrm{24/24h}$ & $2 \mathrm{~g} \mathrm{24/24h}$ & $2 \mathrm{~g} \mathrm{24/24h}$ \\
\hline Azitromicina VO 500mg & 5 dias & $500 \mathrm{mg} 24 / 24 \mathrm{~h}$ & $500 \mathrm{mg} 24 / 24 \mathrm{~h}$ & $500 \mathrm{mg} \mathrm{24/24h}$ \\
\hline Oseltamivir VO 75mg & 5 dias & $75 \mathrm{mg} \mathrm{12/12h}$ & $75 \mathrm{mg} \mathrm{12/12h}$ & $75 \mathrm{mg} \mathrm{12/12h}$ \\
\hline Ivermectina VO 6mg & 3 dias & $6 \mathrm{mg} / 30 \mathrm{~kg} \mathrm{24/24h}$ & $\begin{array}{c}6 \mathrm{mg} / 30 \mathrm{~kg} \\
24 / 24 \mathrm{~h}\end{array}$ & $\begin{array}{c}6 \mathrm{mg} / 30 \mathrm{~kg} \\
24 / 24 \mathrm{~h}\end{array}$ \\
\hline Metilprednisolona EV 500mg & 3 dias & - & $80 \mathrm{mg}$ & $\begin{array}{c}\mathrm{D} 0125 \mathrm{mg} \\
\mathrm{D} 1-\mathrm{D} 380 \mathrm{mg}\end{array}$ \\
\hline
\end{tabular}

Legenda: VO: via oral; SC: subcutânea; EV: endovenosa; g: gramas; mg: miligramas; UI: Unidades Internacionais; h: horas; D0: dose inicial; D1-D3: primeiro ao terceiro dia de tratamento; amp: ampola. ${ }^{\mathrm{a} O}$ Oseltamivir somente era usado até a confirmação do diagnóstico de COVID-19 ou caso não tenha se vacinado para o Influenza. Fonte: Braúna CC, et al., 2020.

Quanto ao custo diário de cada medicamento e a sua porcentagem em relação ao custo diário do tratamento previsto na Tabela SIGTAP [Sistema de Gerenciamento da Tabela de Procedimentos, Medicamentos e OPM (Órteses, próteses e meios auxiliares de locomoção) do SUS (Sistema Único de Saúde)] (BRASIL, 2020), os medicamentos que representaram a maior parte deste valor, caso utilizados, foram a ceftriaxona $R \$ 77,00(32,19 \%)$, omeprazol $R \$ 28,80(12,04 \%)$, heparina [Fases 1 e $2 a \mathrm{R} \$ 32,19$ $(13,46 \%)$ - Fase $2 b \mathrm{R} \$ 64,38(26,91 \%)]$ e a metilprednisolona $R \$ 44,80$ (18,73\%). Os medicamentos off-label por sua vez consumiriam $1,93 \%$ do valor, com a ivermectina custando $R \$ 2,00(0,80 \%)$ e a hidroxicloroquina $R \$ 2,72(1,13 \%)$. O oseltamivir, usado somente em caso de suspeita de influenza, representaria $R \$ 8,00$ $(3,34 \%)$. Assim, o custo total da terapia farmacológica (caso o paciente utilize todos os medicamentos previstos no protocolo da instituição, com exceção do oseltamivir) por fase da infecção foi: fase 1 - $R \$ 155,71$, fase $2 A-R \$ 200,51$ e fase $2 B-R \$ 232,70$ (Tabela 2). 
Tabela 2 - Custo diário do tratamento medicamentoso utilizado para COVID-19 no hospital de campanha estudado em diferentes fases da COVID-19.

\begin{tabular}{|c|c|c|c|c|}
\hline \multirow{2}{*}{ Medicamento } & \multirow{2}{*}{$\begin{array}{c}\text { Regime } \\
\text { terapêutico }\end{array}$} & \multicolumn{3}{|c|}{ Custo diário } \\
\hline & & Fase 1 & Fase 2A & Fase 2B \\
\hline Heparina SC 5000Ul/0,25mL & $5000 \mathrm{UI} 8 / 8 \mathrm{~h}$ & $\mathrm{R} \$ 32,19$ & $\mathrm{R} \$ 32,19$ & $\mathrm{R} \$ 64,38$ \\
\hline Omeprazol EV 40mg & $40 \mathrm{mg} \mathrm{24/24h}$ & $\mathrm{R} \$ 28,80$ & $\mathrm{R} \$ 28,80$ & $\mathrm{R} \$ 28,80$ \\
\hline Hidroxicloroquina VO 400mg & $400 \mathrm{mg} \mathrm{12/12h}$ & $\mathrm{R} \$ 2,72$ & $\mathrm{R} \$ 2,72$ & $\mathrm{R} \$ 2,72$ \\
\hline Ceftriaxona EV 1g & $2 \mathrm{~g} \mathrm{24/24h}$ & $\mathrm{R} \$ 77,00$ & $\mathrm{R} \$ 77,00$ & $\mathrm{R} \$ 77,00$ \\
\hline Azitromicina VO 500mg & $500 \mathrm{mg} 24 / 24 \mathrm{~h}$ & $R \$ 5,00$ & $R \$ 5,00$ & $\mathrm{R} \$ 5,00$ \\
\hline Oseltamivir ${ }^{\mathrm{b}}$ VO $75 \mathrm{mg}$ & $75 \mathrm{mg} \mathrm{12/12 \textrm {h }}$ & $\mathrm{R} \$ 8,00$ & $\mathrm{R} \$ 8,00$ & $\mathrm{R} \$ 8,00$ \\
\hline Ivermectina VO 6mg & $6 \mathrm{mg} / 30 \mathrm{~kg} \mathrm{24/24h}$ & $R \$ 2,00$ & $\mathrm{R} \$ 2,00$ & $\mathrm{R} \$ 2,00$ \\
\hline Metilprednisolona EV 500mg & Variável & - & $\mathrm{R} \$ 44,80$ & $\mathrm{R} \$ 44,80$ \\
\hline $\begin{array}{l}\text { Custo total máximo do tratamento } \\
\text { medicamentoso }\end{array}$ & - & $R \$ 155,71$ & $R \$ 200,51$ & $\mathrm{R} \$ 232,70$ \\
\hline
\end{tabular}

Legenda: VO: via oral; SC: subcutânea; EV: endovenosa; g: gramas; mg: miligramas; UI: Unidades Internacionais; h: horas; D0: dose inicial; D1-D3: primeiro ao terceiro dia de tratamento; amp: ampola. ${ }^{\text {PPara }}$ a fase $2 \mathrm{~B}$ a dose é de $10000 \mathrm{UI} 8 / 8 \mathrm{~h}$. bO Oseltamivir somente era usado até a confirmação do diagnóstico de COVID-19 ou caso não tenha se vacinado para o Influenza; bSupondo um paciente adulto de 60kg; OBS: Cotação do dólar frente ao real durante o período de funcionamento do hospital estudado, US\$1,0 $=R \$$ 5,2675 de acordo com o IPEA - Instituto de Pesquisa Econômica Aplicada. Fonte: Braúna CC, et al., 2020.

O Quadro 1 apresenta a classificação dos medicamentos com indicação prevista em bula (label) ou indicação não prevista em bula (off-label). O texto marcado em negrito destaca o(s) motivo(s) que levaram o medicamento a ser considerado como uso label na infecção primaria por COVID-19. Todavia, a hidroxicloroquina e a ivermectina foram consideradas como uso off-label, tendo em vista que seu uso não possuía justificativa plausível em relação a sintomatologia apresentada na infecção por SARS-CoV-2.

Quadro 1 - Medicamentos propostos, suas indicações e tipo de uso segundo informações do bulário eletrônico da ANVISA e Drugs.com.

\begin{tabular}{|c|c|c|}
\hline Medicamento & Indicação & $\begin{array}{l}\text { Tipo de } \\
\text { uso }\end{array}$ \\
\hline Heparina & $\begin{array}{l}\text { Profilaxia e tratamento de distúrbios tromboembólicos; prevenção da } \\
\text { coagulação em cirurgia arterial e cardíaca; como anticoagulante para } \\
\text { transfusões de sangue, circulação extracorpórea e procedimentos de } \\
\text { diálise. }\end{array}$ & Label \\
\hline Omeprazol & $\begin{array}{c}\text { Úlcera péptica gástrica, ou duodenal; esofagite de refluxo; síndrome de } \\
\text { Zollinger-Ellison; profilaxia de aspiração de conteúdo gástrico durante a } \\
\text { anestesia geral em pacientes de risco. } \\
\text { Proteção do trato gastrointestinal superior em pacientes seriamente } \\
\text { doentes. }\end{array}$ & Label \\
\hline Hidroxicloroquina & Lúpus Eritematoso, malária, artrite reumatoide. & Off-label \\
\hline Ceftriaxona & $\begin{array}{c}\text { Infecções por microrganismos sensíveis a ceftriaxona em locais de } \\
\text { penetração adequada dela. } \\
\text { Profilaxia perioperatória de infecções. }\end{array}$ & Label \\
\hline Azitromicina & $\begin{array}{c}\text { Infecções por microrganismos sensíveis a azitromicina em locais de } \\
\text { penetração adequada dela. }\end{array}$ & Label \\
\hline Oseltamivir & Profilaxia ou tratamento de influenza (A ou B). & Label \\
\hline Ivermectina & $\begin{array}{c}\text { Infecções por Strongyloides stercoralis, Onchocerca volvulus, Wuchereria } \\
\text { bancrofti, Ascaris lumbricoides, Sarcoptes scabiei, Pediculus humanus } \\
\text { capitis. }\end{array}$ & Off-label \\
\hline Metilprednisolona & $\begin{array}{l}\text { Disturbios endócrinos, hematologicos e reumáticos; Doenças do colágeno, } \\
\text { do complexo imunológico, dermatológicas, oftálmicas, gastrintestinais, } \\
\text { respiratórias, neoplásicas, estados alérgicos entre outros. }\end{array}$ & Label \\
\hline
\end{tabular}

Fonte: Braúna CC, et al., 2020. 


\section{DISCUSSÃO}

O custo do tratamento farmacológico de uma doença é dependente da sua gravidade e dos sintomas tratados. Siddiqi HK e Mehra MR (2020), propuseram para COVID-19, uma classificação em três estágios: 1 - Inicio da infecção (febre, dor de cabeça, tosse seca); 2 - Fase pulmonar (2a - dificuldade para respirar sem hipóxia, imagens pulmonares com alterações, $2 b$ - (Hipóxia) e; 3 - Fase hiperinflamatória (tempestade de citocinas, IRHA (insuficiência Respiratória Hipoxêmica Aguda), IRA (Insuficiência Respiratória Aguda) e disfunção orgânica múltipla). O hospital estudado desenvolveu seu protocolo visando o atendimento de pacientes nos estágios 1, 2a e 2b da infecção pelo novo coronavírus - COVID-19, os pacientes que evoluíam para o estágio 3 eram transferidos para um local ao qual possuía suporte adequado (Tabela 1).

\section{Financiamento do tratamento}

Uma das principais preocupações no início da pandemia para os gestores foi o gasto público que o COVID19 poderia gerar aos sistemas de saúde ao redor do globo. Em um estudo americano no qual simulou o custo que a pandemia poderia ocasionar. Demostrou que um único caso sintomático de COVID-19 poderia incorrer em um custo direto de em média US\$3.045, considerando somente a fase ativa da doença. Além disso, o artigo destaca que autoridades sanitárias e profissionais de saúde levantaram preocupações de que a demanda de pacientes em hospitais poderia exceder a capacidade existente, o que já aconteceu em diversos locais, e solicitam recursos adicionais e apoio financeiro (BARTSCH, et al., 2020).

No Brasil, o Ministério da Saúde através da Secretaria de Atenção Especializada à Saúde, publicou a portaria №245, de 24 de março de 2020, criando o procedimento "03.03.01.022-3 - TRATAMENTO DE INFECÇÃO PELO NOVO CORONAVIRUS - COVID 19" para a tabela unificada do SIGTAP. De acordo com esta portaria, o valor previsto para o serviço hospitalar equivale à quantia de $R \$ 1195,99$ prevendo uma média de duração da internação de cinco dias, perfazendo assim o valor diário médio de $R \$ 239,20$ para custear o tratamento da infecção por SARS-CoV-2 (BRASIL, 2020).

Entretanto, este valor pode ser insuficiente segundo os valores encontrados em nosso estudo (Tabela 2), visto que o custo máximo diário de acordo com a fase do tratamento medicamentoso de COVID-19 no hospital campanha estudado alcançou na fase $1-R \$ 155,71$; fase $2 a-R \$ 200,51$ e; fase $2 b-R \$ 232,70$. Tais montantes estão envolvidos apenas com os custos diretos (medicamentos) da terapia farmacológica, de COVID-19, não abrangendo custos indiretos com a internação como higienização, alimentação, materiais hospitalares entre outros. Consumindo assim, percentualmente, o tratamento medicamentoso proposto pela instituição de saúde para a infecção por estágio: fase $1-65,10 \%$, fase $2 a-83,83 \%$ e fase $2 b-97,28 \%$ do valor diário médio previsto no procedimento criado pelo MS para o tratamento de COVID 19".

A Planisa, uma empresa de consultoria especializada na área saúde, realizou um estudo em doze hospitais brasileiros (entre públicos, privados e filantrópicos) que atenderam pacientes com COVID-19, no período de abril a maio de 2020. Nesse estudo considerou-se custos diretos e indiretos - gastos com a profissionais de saúde, materiais, medicamentos, EPIs (Equipamentos de Proteção Individual), serviços de apoio e administrativo, entre outros. Os resultados mostraram que o custo médio de diária hospitalar em unidade de internação não crítica com paciente Covid-19 foi $R \$ 1.400$, com taxa de ocupação média de $50 \%$; enquanto em unidades de internação crítica adulto (UTI), o custo/dia médio foi $R \$ 2.452$. É importante ressaltar que houve variações de custos nas diárias hospitalares. Em geral, hospitais que atendem pacientes mais complexos, o custo unitário da diária é maior do que em hospitais que atendem casos mais simples. Neste caso, o consumo de medicamentos e materiais hospitalares são os principais responsáveis no aumento destes custos (PORTAL HOSPITAIS BRASIL, 2020).

Outro estudo realizado em 7 hospitais de referência no tratamento de COVID-19 observou maior consumo em materiais e medicamentos hospitalares para pacientes em unidade de internação não-crítica COVID-19, que girou em torno de $\mathrm{R} \$ 156,00$ por diária, enquanto na unidade de internação não-crítica destinada à pacientes com outras enfermidades, nestes mesmos hospitais, foi de $R \$ 107,00$, portanto, alta de $46 \%$. Na UTI, o custo médio foi $\mathrm{R} \$ 594,56$ para pacientes COVID-19 por diária e $\mathrm{R} \$ 399,02$ para pacientes não COVID19, aumento de 49\% (PORTAL HOSPITAIS BRASIL, 2021). 
Há de se equacionar também o impacto do custo do tratamento da infecção por SARS-CoV-2 do ponto de vista farmacológico e suas evidências terapêuticas, pois um medicamento mal escolhido, além de representar um custo evitável, pode gerar outros custos secundários, associados a eventos adversos, falhas terapêuticas entre outros (BIGUETTI C, et al., 2020; LOVE JS, et al., 2020). Além disso, quando esse gasto público é extrapolado para a escala da máquina estatal, seu impacto pode ser ainda mais danoso visto que, a arrecadação monetária viu-se diminuída frente as medidas de contenção necessárias para mitigar a transmissão do vírus SARS-CoV-2 (BRESSER-PEREIRA LS, 2020).

\section{Escolhas terapêuticas e indicações medicamentosas para o tratamento de COVID-19.}

Diante da pandemia de COVID-19 devemos salientar ainda que desde o final de 2019 vários estudos estão sendo realizados na busca de alternativas terapêuticas para o tratamento da infecção aguda por SARS-CoV2. O diretor geral da OMS, anunciou em março de 2020, a realização de um grande estudo clínico para testar medicamentos com atividade contra SARS-CoV-2 (OMS, 2020a).

Esse estudo possuía o registro de protocolo (NCT04321616) e se propôs a avaliar os medicamentos remdesivir, lopinavir/ritonavir, cloroquina/hidroxicloroquina e interferon beta. No Brasil, a Fiocruz foi parceira nessa iniciativa da OMS (FUNDAÇÃO OSWALDO CRUZ, 2020). Todavia no dia 15 de outubro de 2020, foi anunciado que o grupo de estudo do SOLIDARITY não encontrou evidencias científicas de efeito na mortalidade, necessidade de ventilação mecânica e duração da internação, dos pacientes que usavam os medicamentos supracitados no tratamento da COVID-19 (OMS, 2020b).

Além disso, outros medicamentos foram teorizados como possíveis agentes farmacológicos contra a COVID-19, sendo eles antivirais (LIU F, et al. 2020), corticosteroides (RUSSELL CD, et al. 2020), antiparasitarios (OLIVEIRA HA, et al. 2020), antimaláricos (WANG M, et al. 2020) e antimicrobianos (CORTEGIANI A, et al., 2020; GAUTRET P, et al., 2020). Adicionalmente, muitos dos estudos são baseados no mecanismo de funcionamento dos fármacos, sendo essencialmente teóricos ou em vitro (CORTEGIANI A, et al., 2020; SANDERS JM, et al., 2020; WANG M, et al., 2020). No entanto, não existe até o momento terapia farmacológica sabidamente eficaz contra o COVID-19 (OMS, 2020b) e a desinformação médica pode causar graves danos aos pacientes com COVID-19, sendo por vezes causada por evidencias fracas ou por opiniões profissionais (LOVE JS, et al., 2020).

Uma controvérsia a ser discutida é o uso da hidroxicloroquina e da ivermectina (uso off-labe/), no tratamento da infecção por SARS-CoV-2 (Quadro 1). A cloroquina e seu análogo a hidroxicloroquina foram drogas propostas para surtos anteriores de outras doenças provocadas por coronavírus (SARS-CoV e MERSCoV), razão pela qual justifica-se o interesse inicial como possíveis formas de tratamentos para o SARS-CoV2 (AL-BARI MAA, 2015; IMOTO AM, et al., 2020; VINCENT MJ, et al., 2005; ZHOU D, et al., 2020).

Entretanto, um estudo alertou que o uso da hidroxicloroquina em pneumonia associada ao COVID-19, ocasiona o aumento do prolongamento do intervalo QTc, risco que aumenta com a associação da azitromicina (MERCURO NJ, et al., 2020). As Diretrizes Brasileiras para o Tratamento Farmacológico da Covid-19 preconizam que as evidências disponíveis não sugerem benefício clinicamente significativo do tratamento com hidroxicloroquina ou com cloroquina. O painel de recomendações também entendeu que as evidências disponíveis do tratamento com Hidroxicloroquina $\mathrm{HCQ}$ ou com Cloroquina (CQ) em associação com a azitromicina (AZ) não sugerem benefícios clinicamente significativos. Houve entendimento de que o risco de eventos adversos cardiovasculares é moderado, em especial de arritmias, sendo potencializado com a associação de $\mathrm{HCQ} / \mathrm{CQ}$ com $\mathrm{AZ}$, necessitando maiores cuidados em relação a esses eventos adversos (FALAVIGNA M, et al., 2020).

Já em relação à ivermectina, este é um fármaco antiparasitário utilizado a cerca de 40 anos. Todavia recentemente, foi demonstrado que ela possui atividade antiviral para uma grande quantidade de vírus como o da Dengue, HIV, Influenza e encefalite equina venezuelana. Seu possível mecanismo de ação deve-se a dependência de diferentes vírus de RNA pela IMPa/ß1 durante a infecção. O SARS-CoV-2 possui semelhança inegável com o SARS-CoV, este último vírus também possui dependência para o IMPa/ $\beta 1$ durante o ciclo da infecção para o fechamento nucleocitoplasmático. 
No entanto, ainda não existem evidências robustas que embasem seu emprego no tratamento da COVID19, pois apesar de apresentar atividade antiviral in vitro para o SARS-CoV-2 (CALY L, et al., 2020). Do ponto de vista farmacocinético, nas condições simuladas pelo artigo de Momekov G e Momekova D (2020) é impraticável alcançar as concentrações requeridas na simulação in vitro, pois de acordo com eles caso a concentração fosse extrapolada para um humano, a dose necessária para alcançar a concentração sérica pretendida ultrapassaria a janela terapêutica do medicamento, podendo assim apresentar mais efeitos tóxicos do que terapêuticos para o indivíduo.

Outros três medicamentos que mereceram destaque em nosso estudo foram os antibióticos - Ceftriaxona e Azitromicina - e o antiviral Oseltamivir (Quadro 1). Apesar de tais fármacos antibióticos não possuírem atividade comprovada contra o SARS-CoV-2, a coinfecção bacteriana o surgimento de coinfecções em indivíduos com processos inflamatórios é uma grande preocupação, ocorrendo em até $50 \%$ dos pacientes que não sobreviveram a infecção (ZHOU D, et al., 2020).

No estudo de Lai CC, et al. (2020), uma revisão sobre a coinfecção em pacientes com COVID-19, mostrou que diversas bactérias, fungos e vírus podem associar-se a infecção por SARS-CoV-2 agravando o quadro clínico dos pacientes. No entanto, das recomendações avaliadas pelo estudo de Lai et al. sobre medicamento sem atividade para SARS-CoV-2 no tratamento infecções secundarias na COVID-19, mantem-se as recomendações sobre o uso empírico, descalonamento e uso racional de antimicrobianos. Em vista disso o protocolo desenvolvido pelo hospital campanha estudado para tratamento da COVID-19 poderia ter destacado que o uso de antimicrobianos na COVID-19, somente está indicado quando existe a suspeita de coinfecção bacteriana, a fim de reforçar o uso racional dos antimicrobianos.

É importante ressaltar que o Oseltamivir - de acordo com o Protocolo de tratamento de influenza 2017 do Ministério da Saúde Brasileiro - está indicado em caso de suspeita de síndrome gripal por decisão baseada em somente julgamento clínico, independentemente da situação vacinal, caso exista condições ou fatores de riscos para complicações, como gravidez, idade inferior a 5 anos ou maior que 60 anos, população indígena em situação de isolamento, comorbidades e SRAG (Síndrome Respiratória Aguda Grave). Sendo sua dose usual para adultos com função renal adequada de $75 \mathrm{mg}$, duas vezes ao dia, por cinco dias (BRASIL, 2017).

SRAG é o SARS sigla em inglês que significa Severe Acute Respiratory Syndrome, o principal agravo da infecção por SARS-CoV-2. Além disso boa parte das comorbidade que levam a um pior prognostico da infecção sintomática por influenza (BRASIL, 2017) levam a mesma piora no caso da COVID-19 (WU C, et al., 2020). Sendo assim a inclusão no protocolo mostra-se essencial e bem embasada.

\section{CONCLUSÃO}

Uma estratégia promissora para tratamento da COVID-19 pareceu ser o uso de medicamentos que não possuem eficácia comprovada visto que alguns estudos analisados apontaram para o uso destes como possível terapia farmacológica contra a infecção. Entretanto, o protocolo medicamentoso para COVID-19 utilizado no hospital campanha estudado, mostrou-se bem oneroso frente ao financiamento pretendido. Espera-se que avaliações farmacoeconômicas como a realizada por esse estudo permitam o melhor desenho de novas versões do protocolo e otimização de gastos no tratamento.

\section{REFERÊNCIAS}

1. AL-BARI MAA. Chloroquine analogues in drug discovery: new directions of uses, mechanisms of actions and toxic manifestations from malaria to multifarious diseases. J Antimicrob Chemother, 2015;70(6):1608-21.

2. ANVISA. Agência Nacional de Vigilância Sanitária. 2020a. PREÇOS MÁXIMOS DE MEDICAMENTOS POR PRINCÍPIO ATIVO. CMED - Câmara de Regulação do Mercado de Medicamentos. Disponível em: http://portal.anvisa.gov.br/documents/374947/6015130/LISTA_CONFORMIDADE_2020_09_v1.pdf/0e0af67c-ca314712-91b0-eb1f97593807. Acesso em: 11 set. 2020.

3. ANVISA. Agência Nacional de Vigilância Sanitária. 2020b. Bulário Eletrônico. Disponível em: http://www.anvisa.gov.br/datavisa/fila_bula/index.asp. Acesso em: 11 set. 2020.

4. BARBIRATO DS, et al. Estado atual do uso da cloroquina/hidroxicloroquina no tratamento da COVID-19: uma Overview. Braz. J. of Develop, 2020;6(8):62988-62997.

5. BARTSCH SM, et al. The Potential Health Care Costs And Resource Use Associated With COVID-19 In The United States. Health Affairs, v. 39, n. 6, p. 927-935, 1 jun. 2020. 
6. BIGUETTI C, et al. Primum non nocere - Are chloroquine and hydroxychloroquine safe prophylactic/treatment options for SARS-CoV-2 (covid-19)? Rev Saude Publica, 2020;54:68.

7. BRASIL. Ministério da Saúde. Secretaria de Vigilância em Saúde. Departamento de Vigilância das Doenças Transmissíveis. Protocolo de Tratamento de Influenza 2017. Brasília: Ministério da Saúde, 2027. 51p.

8. BRASIL. Ministério da Saúde. Secretaria de Atenção Especializada à Saúde. Portaria № 245, de 24 de março 2020. Brasília: Ministério da Saúde, 2020.

9. BRESSER-PEREIRA LC. Financiamento da Covid-19, inflação e restrição fiscal. Brazilian J Polit Econ, 2020;40(4):604-21.

10. CALY L, et al. The FDA-approved drug ivermectin inhibits the replication of SARS-CoV-2 in vitro. Antiviral Res, 2020;178:104787.

11. CORTEGIANI A, et al. A systematic review on the efficacy and safety of chloroquine for the treatment of COVID-19. J Crit Care, 2020;57:279-83.

12. DRUGS.COM. 2020. In: Drugs.com. Disponível em: https://www.drugs.com/. Acesso em: 11 set. 2020.

13. FALAVIGNA M, et al. Guidelines for the pharmacological treatment of COVID-19. The task force/consensus guideline of the Brazilian Association of Intensive Care Medicine, the Brazilian Society of Infectious Diseases and the Brazilian Society of Pulmonology and Tisiology. Rev Bras Ter Intensiva, 2020;32(2).

14. FIOCRUZ. Fundação Oswaldo Cruz. 2020. Fiocruz lidera no Brasil ensaio clínico «Solidarity» (Solidariedade) da OMS. Disponível em: https://portal.fiocruz.br/noticia/fiocruz-lidera-no-brasil-ensaio-clinico-solidarity-solidariedade-da-oms. Acesso em: 3 set. 2020.

15. GAUTRET P, et al. Hydroxychloroquine and azithromycin as a treatment of COVID-19: results of an open-label nonrandomized clinical trial. Int J Antimicrob Agents, 2020;56(1):105949.

16. HUGG-UNIRIO. 2020. In: Protocolo para manejo clínico, diagnóstico, avaliação e tratamento da COVID 19.

17. IMOTO AM, et al. Cloroquina e Hidroxicloroquina no tratamento da COVID-19: Sumário de Evidências TT - Chloroquine and Hydroxychloroquine in the treatment of COVID-19: Summary of Evidence. Comun em ciências da saúde, 2020;31(suppl 1):17-30.

18. LAI CC, et al. Co-infections among patients with COVID-19: The need for combination therapy with non-anti-SARSCoV-2 agents? J Microbiol Immunol Infect, 2020;53(4):505-12.

19. LIU F, et al. Patients of COVID-19 may benefit from sustained Lopinavir-combined regimen and the increase of Eosinophil may predict the outcome of COVID-19 progression. Int J Infect Dis, 2020;95:183-91.

20. LOVE JS, et al. The Parallel Pandemic: Medical Misinformation and COVID-19. J Gen Intern Med, 2020;35(8):24356.

21. MERCURO NJ, et al. Risk of QT Interval Prolongation Associated With Use of Hydroxychloroquine With or Without Concomitant Azithromycin Among Hospitalized Patients Testing Positive for Coronavirus Disease 2019 (COVID-19). JAMA Cardiol, 2020;5(9):1036.

22. MOMEKOV G, MOMEKOVA D. Ivermectin as a potential COVID-19 treatment from the pharmacokinetic point of view: antiviral levels are not likely attainable with known dosing regimens. Biotechnol Biotechnol Equip, 2020 Jan $1 ; 34(1): 469-74$.

23. OLIVEIRA HA, et al. In: Ivermectina para o tratamento de pacientes com COVID-19. Revisão sistemática rápida, 2020.

24. OMS. 2020. In: "Solidarity" clinical trial for COVID-19 treatments.

25. OMS. 2020. In: WHO Director-General's opening remarks at the media briefing on COVID-19.

26. PORTAL HOSPITAIS BRASIL. 2020. In: Custo hospitalar da Covid-19 para cada 1,37 mi de infectados é de cerca de $\mathrm{R} \$ 3,1$ bi.

27. PORTAL HOSPITAIS BRASIL. 2021. In: Covid-19: Custo mediano de diária em UTI é de R $\$ 2.234,00$.

28. RUSSELL CD, et al. Clinical evidence does not support corticosteroid treatment for 2019-nCoV lung injury. Lancet (London, England), 2020;395(10223):473-5.

29. SANDERS JM, et al. Pharmacologic Treatments for Coronavirus Disease 2019 (COVID-19): A Review. JAMA - Journal of the American Medical Association, 2020;323(18)1824-36.

30. SHANG L, et al. On the use of corticosteroids for 2019-nCoV pneumonia. Lancet (London, England), 2020;395(10225):683-4.

31. SHEAHAN TP, et al. Broad-spectrum antiviral GS-5734 inhibits both epidemic and zoonotic coronaviruses. Sci Transl Med, 2017;9(396):eaal3653.

32. SHEREEN MA, et al. COVID-19 infection: Origin, transmission, and characteristics of human coronaviruses. J Adv Res,2020;24:91-98.

33. SIDDIQI HK, MEHRA MR. COVID-19 illness in native and immunosuppressed states: A clinical-therapeutic staging proposal. J Hear Lung Transplant, 2020;39(5):405-7.

34. TILLU G, et al. Public Health Approach of Ayurveda and Yoga for COVID-19 Prophylaxis. J Altern Complement Med, 2020;26(5):360-4.

35. VINCENT MJ, et al. Chloroquine is a potent inhibitor of SARS coronavirus infection and spread. Virol J, 2005;2:69.

36. WALLEY T, HAYCOX A. Pharmacoeconomics: basic concepts and terminology. Br J Clin Pharmacol, 1997;43(4):3438

37. WANG M, et al. Remdesivir and chloroquine effectively inhibit the recently emerged novel coronavirus (2019-nCoV) in vitro. Cell Res, 2020;30(3):269-71.

38. WU C, et al. Risk Factors Associated With Acute Respiratory Distress Syndrome and Death in Patients With Coronavirus Disease 2019 Pneumonia in Wuhan, China. JAMA Intern Med, 2020;180(7):934.

39. ZHOU D, et al. COVID-19: a recommendation to examine the effect of hydroxychloroquine in preventing infection and progression. J Antimicrob Chemother, 2020;75(7):1667-70.

40. ZHOU F, et al. Clinical course and risk factors for mortality of adult inpatients with COVID-19 in Wuhan, China: a retrospective cohort study. Lancet, 2020;395(10229):1054-62. 